\title{
Mean-Conditional Value-at-Risk Optimal Energy Storage Operation in the Presence of Transaction Costs
}

\author{
Somayeh Moazeni, Member, IEEE, Warren B. Powell, Member, IEEE, Amir H. Hajimiragha, Senior \\ Member, IEEE,
}

\begin{abstract}
This paper addresses the formulation and solution of an optimal energy storage management problem under risk consideration and transaction costs of trading energy with the power grid. The price evolves as a stochastic process, capable of correctly explaining the seasonality effects as well as the tail fatness and spikiness in its distribution. Transaction costs capture the price impact of the storage operation on the electricity spot price. A risk analysis of an optimal risk neutral deterministic policy as well as the simple myopic policy indicates that the realized operational cost may notably differ from the expected cost by a considerable probability. This difference suggests that we need to consider risk. Using the downside risk measure of Conditional Value-at-Risk, an optimal risk averse conversion and transmission strategy, among the grid, the renewable power generation source, and an energy storage is proposed to fully satisfy the electricity demand and minimize the expected operational cost as well as the risk. Our numerical study using data from NYISO demonstrates the impacts of risk consideration and the transaction cost parameters on the optimal strategy structure, its expected cost, and its risk.
\end{abstract}

Index Terms-Energy storage, Risk, CVaR optimization.

\section{NOMENCLATURE}

$x_{t}^{I J}$ Amount of energy transferred from unit $I$ to unit $J$ at time step $t$ [MWh].

$\beta$ Confidence level of the downside risk measure [-].

$\omega_{\mathbb{E}}, \omega_{\rho}$ Risk aversion parameters [-].

$\alpha_{t}^{G I}$ Transaction costs of exchanging energy from grid to unit $I$ [\$/MWh].

$\alpha_{t}^{I G}$ Transaction costs of exchanging energy from unit $I$ to grid $[\$ / \mathrm{MWh}]$.

$\gamma_{\Delta t}$ Rate of energy loss over a time interval of length $\Delta t[-]$.

$\eta^{\mathrm{C}}$ Charging efficiency [-].

$\eta^{\mathrm{D}}$ Discharging efficiency [-].

$S^{\text {cap }}$ Maximum capacity of the storage device [MWh].

$\Delta S^{\mathrm{C}}$ Maximum charging rate [-].

$\Delta S^{D}$ Maximum discharging rate [-].

$S^{\max }$ Maximum acceptable charge level [-].

$S^{\text {min }}$ Minimum acceptable charge level [-].

$M$ Number of Monte Carlo simulations.

$\tilde{P}_{t}$ Energy price over the $t^{\text {th }}$ time step [ $\$ / \mathrm{MWh}$ ].

$E_{t}$ Energy output from the wind turbine over the $t^{\text {th }}$ time step [MWh].

S. Moazeni and W. B. Powell are with the Department of Operations Research and Financial Engineering, Princeton University, Princeton, NJ, 08544 USA, e-mails: somayeh@princeton.edu and powell@princeton.edu (see http://www.castlelab.princeton.edu/). A. H. Hajimiragha is with GE Digital Energy, Markham, ON, Canada L6C 0M1, e-mail: ahajimir@ieee.org.
$D_{t}$ Energy demand over the $t^{\text {th }}$ time step [MWh].

$T$ Planning horizon.

$T_{N}$ Number of time steps.

\section{INTRODUCTION}

$\mathbf{E}$ NERGY storage systems are becoming indispensable components of existing and future electricity grids by improving the grid economy, reliability, and stability [1], [2]. Different types of energy storage technologies with various response times can serve power system requirements in different time scales [3]. Thus, over a short time span (i.e., seconds to minutes), fast-response energy storage systems can offset the short-term fluctuations in demand and intermittent renewable power generation, and thereby improve the dynamic performance of the power systems. Over a long time span (i.e., hours to days), energy storage systems can potentially offer significant economic advantages by shifting the energy through absorbing surplus or inexpensive energy during offpeak hours, and then discharging it during on-peak hours, when electricity prices are typically high. In view of these considerations, grid-scale storage has drawn the attention of utilities throughout the world as a means to address many challenges they are dealing with, especially at present, when the penetration of intermittent and inflexible renewable power sources is on the rise. Grid-scale energy storage can provide a range of appealing benefits for the utilities, which include but are not limited to load leveling and peak shaving, smoothing power fluctuations, relieving transmission congestion, and deferring network upgrades [4]. However, an effective use of storage technology in energy and power systems is impossible without efficient operation management, especially when their application over a long time period is considered; this is the main theme of the present study.

Variations of the storage operation management problem have been previously studied in the literature. Three control policies and their corresponding (expected) gained revenues are analyzed in [5], assuming probabilistic models for load and wind in the presence of the local network voltage perturbations. The sizing and management of a hydro pumping storage in an island power system with renewable penetration and deterministic generation cost are studied in [6]. The goal is to minimize the (expected) operation and annualized installation costs over all scenarios of wind, hydropower production, and load. Maximization of the (expected) market 
profit of a wind farm and a hydro pumped storage owner over a finite horizon to comply with his commitments in the market is discussed in [7]. The problem is formulated as a two-stage stochastic programming problem. An optimal bidding strategy in a day-ahead market, to maximize the (expected) discounted rewards from the bidding process for the owner of a hybrid system of renewable power generation and energy storage is investigated in [8]. This problem is modeled as a continuous-state Markov decision process and solved using approximate dynamic programming. A similar problem of maximizing the total discounted revenue of a storage owner when trading with the grid over a finite horizon and in the absence of any demand is studied in [9], where a simulation-based numerical method is proposed. An optimal energy commitment policy for the owner of a wind farm and a storage to maximize the (expected) revenue in an infinite horizon is investigated in [10], where both electricity price and wind power supply evolve as stochastic processes. In [11], a setting that involves conventional generation with a real time ex-ante price, renewable generation with a zero marginal cost, and an energy storage is considered. The goal is to fully serve elastic demand as well as to minimize the (average) cost of used conventional generation and investment in storage. The authors formulate the problem as a discrete time (average) cost stochastic dynamic programming problem over an infinite horizon and establish the existence of an optimal stationary policy. The optimal policy suggests to store when there is excess generation (over demand) and to extract energy from the storage device if there is excess demand (over generation). A virtual power plant consisting of an intermittent source, a storage facility, and a dispatchable power plant is considered in [12]. The virtual power plant sells and purchases electricity in both the day-ahead and the balancing markets in order to maximize the expected profit. The authors in [13] consider a weekly self-scheduling of a virtual power plant composed of intermittent renewable sources, storage system and a conventional power plant. The virtual power plant seeks to maximize its expected overall profit, while fulfilling its long-term bilateral contracts.

The vast majority of the literature on operation management of energy storage devices, including the aforementioned studies, minimize expected costs over the planning horizon, which implies that the controller is risk neutral. This ignores the reality that energy prices are highly volatile, exhibit pervasive spikes of extraordinary magnitude, and follow a fat-tailed distribution, e.g., see [14]. For example, the analysis in [15] on the daily average price data from April 1, 1998 to March 31, 2002 for the PJM Western Hub reports a skewness of 8.0 and kurtosis of 80.8 , which clearly confirm the fat tail behavior in the electricity price distribution. The spikes may be due to sudden changes in the slope of the supply curve at the grid level. Indeed, price risk is the most significant risk exposure in energy systems [16]. Therefore, merely optimizing the expected cost (or revenue) is not sufficient and realistic risk considerations must be incorporated when looking for an efficient system operation strategy.

Here, we study the finite time horizon operation management of an energy system including a storage device, a time-variant renewable power generation source, and a load unit. The system owner is also responsible for satisfying the demand, with the capacity to trade power with the grid. The goal is to minimize the total operational cost and price risk exposure of the system. This model, although simple, is a building block in many advanced electric power systems.

The storage device is characterized by storage capacity limitations, conversion and dissipation losses, and maximum rates of conversion. Such a model encompasses various types of storage technologies and explains the essential storage behavior that arises in practice. Since wind power is one of the fastest-growing renewable energy sources (e.g., [17], [18]), this paper considers it for the energy generation. The timevarying load and electricity spot prices are reasonably explained by seasonality effects. The electricity price is assumed to evolve based on a mean reverting jump diffusion stochastic process, which is capable of addressing the main characteristics of energy prices. We calibrate the model to data for the New York Independent System Operator (NYISO).

First, we demonstrate that the realized operational cost of an optimal risk neutral deterministic policy as well as the myopic policy can differ significantly from the expected cost, with a considerable probability. For example, in almost $20 \%$ of the cases, the actual realized operational cost of the optimal risk neutral operation policy deviates from its expected cost by around $18 \%$ (see the second row of Table I-Policy ${ }_{E}$ ). In $10 \%$ of the cases, the actual realized operational cost of this policy deviates from its expected cost by around $29 \%$ (see the third row of Table I-Policy ${ }_{E}$ ). The deviation from expected costs becomes more significant as the confidence level increases. This clearly indicates the relevance of risk consideration in the context of storage operation management.

Second, we suggest incorporating a downside risk measure, such as the Conditional Value-at-Risk (CVaR) in the objective function to properly model aversion to the (price) risk. The CVaR risk measure enjoys nice properties such as convexity [19] and has been widely used to model risk aversion in various industries, such as finance (e.g., [20], [21]) or electricity markets (e.g., [22], [23]). An efficient method to find a mean-CVaR optimal (deterministic) system operation policy is then developed. The approach relies upon a continuously differentiable smoothing function to approximate the non-differentiability from CVaR definition and an augmented Lagrangian method.

We then analyze the structure of the computed risk averse system operation strategy and compare it with the optimal risk neutral strategy. The optimal risk neutral strategy has a dualthreshold pattern; it keeps the storage level at its maximum when the gradient of the expected cost is positive and remains at the minimum charging status when the expected cost is decreasing over periods (see Fig. 5). However, the optimal risk averse strategy suggests interior levels and more frequent charging-discharging conversions. The frequency of changes in the charge level increases, as the CVaR confidence level approaches one (see the plots in Fig. 6). Furthermore, while the incurred expected operational cost of the optimal risk averse policy is only about $1 \%$ more than the expected cost of the optimal risk neutral policy, by adopting the optimal risk neutral 
operation policy the risk to contend with could be up to $13 \%$ more than what the risk averse strategy would have yielded (see Table VII). For example, the expected shortfall at $95 \%$ level of the optimal risk neutral policy is $9.99 \%$ more than that of the optimal risk averse storage operation policy.

In addition to risk consideration in the storage operation management problem, we introduce transaction costs into the model which include the cost of trading with the grid through an intermediate entity such as a utility or a power management company. The literature on the problem typically assumes that the decision maker is price taker and is a small player in a large market, i.e., the storage size is small or any demand shortfall can be made up by buying from the grid at the current spot price; see [6] or [9] for an explicit discussion. These assumptions ignore the storage operation's impact on the electricity spot prices. This concern is also briefly pointed out in section 7 of [9]. The introduced transaction cost parameters in the definition of stage costs can explain effects of the storage operation and trading activities with the grid on the electricity spot prices. We study sensitivity of the structure of the optimal operation strategy, its cost, and its risk to these parameters.

The remainder of the paper is structured as follows. Section II presents the optimization model of the energy storage operation management problem studied in this paper. Risk analysis of the optimal risk neutral policy and myopic policy using the most popular downside risk measure is discussed in Section III. A computationally tractable approach to compute an optimal risk averse energy storage policy under CVaR is provided in Section IV. The results of our numerical experiment, policy analysis, and sensitivity analysis are presented in Section V. Concluding remarks are given in Section VI.

\section{Energy Storage Management Problem}

Consider an energy system involving an electrical grid, a renewable power generation source (e.g., wind), an energy storage, and a demand, as shown in Fig. 1. The owner of the power generator and storage is also responsible to satisfy the demand for a finite planning horizon starting at time 0 and ending at time $T$. The time horizon $[0, T)$ is discretized into $T_{N}$ intervals of length $\Delta t=\frac{T}{T_{N}}$. At each of the $T_{N}$ time steps, the load is served by either the wind power, energy from the storage, or from the grid.

At the $t^{\text {th }}$ time step, the decision is on the following seven dimensional vector:

$$
X_{t} \stackrel{\text { def }}{=}\left(x_{t}^{W D}, x_{t}^{G D}, x_{t}^{W G}, x_{t}^{W S}, x_{t}^{G S}, x_{t}^{S G}, x_{t}^{S D}\right)
$$

for $t=0,1, \cdots, T_{N}-1$. Here, $x_{t}^{I J}$ denotes the amount of energy transferred from unit $I$ to unit $J$ at time step $t$. The superscript $W$ stands for power generator (e.g., wind), $D$ for demand, $S$ for storage, and $G$ for grid. All of these variables are non-negative.

Let $S_{t}$, referred to as the storage level, denote the fraction of the storage which is full at the $t^{\text {th }}$ time step. We assume $S_{0}$ is given and $S_{t}$ evolves according to:

$$
S_{t+1}=\left(1-\gamma_{\Delta t}\right) S_{t}+\frac{\eta^{\mathrm{C}}\left(x_{t}^{G S}+x_{t}^{W S}\right)-\left(x_{t}^{S D}+x_{t}^{S G}\right)}{S^{\mathrm{cap}}},
$$

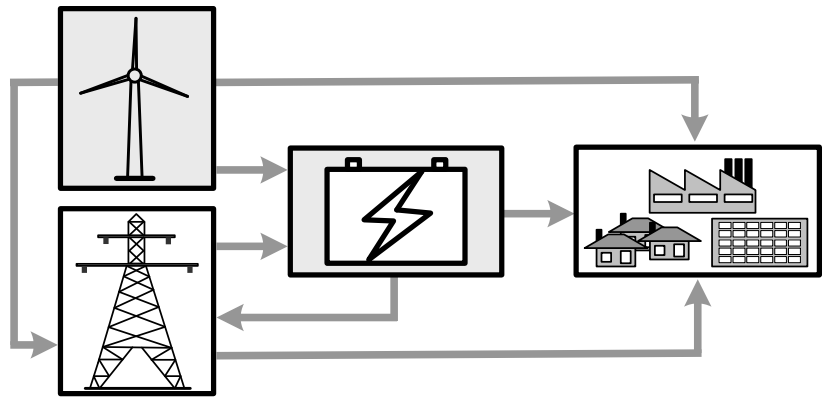

Fig. 1: Energy system diagram.

for $t=0,1, \cdots, T_{N}-1$. Here, $S^{\text {cap }}$ is the maximum capacity of the storage device. The constants $0 \leq \gamma_{\Delta t} \leq 1$ and $0<$ $\eta^{\mathrm{C}} \leq 1$ represent the fraction of the storage charge level which is lost during the time interval of length $\Delta t$ and the fraction of $1 \mathrm{MWh}$ increase in the storage charge level as a result of 1-hour charging at the $1 \mathrm{MW}$ input power, respectively.

By implementing $X_{t}$ at the $t^{\text {th }}$ time step, the system owner incurs the following cost (in dollar value) through exchanging energy with the grid:

$$
\begin{array}{r}
C_{t}\left(\tilde{\mathbf{P}}_{t}, X_{t}\right) \stackrel{\text { def }}{=}\left(\tilde{P}_{t}^{G S}+\alpha_{t}^{G S}\right) x_{t}^{G S}+\left(\tilde{P}_{t}^{G D}+\alpha_{t}^{G D}\right) x_{t}^{G D} \\
-\eta^{D}\left(\tilde{P}_{t}^{S G}-\alpha_{t}^{S G}\right) x_{t}^{S G}-\left(\tilde{P}_{t}^{W G}-\alpha_{t}^{W G}\right) x_{t}^{W G}
\end{array}
$$

where, $\tilde{\mathbf{P}}_{t}=\left(\tilde{P}_{t}^{G S}, \tilde{P}_{t}^{G D}, \tilde{P}_{t}^{S G}, \tilde{P}_{t}^{W G}\right)^{\top}$ refers to the electricity price, and $0<\eta^{D} \leq 1$ indicates discharging efficiency. The cost function in equation (3) assumes that the grid pays for the amount of energy which is received, i.e., $\eta^{D} x_{t}^{S G}$, not the amount $x_{t}^{S G}$ discharged from the storage.

The non-negative constants $\alpha_{t}^{G S}, \alpha_{t}^{G D}, \alpha_{t}^{S G}, \alpha_{t}^{W G}$ in equation (3) explain transaction costs of exchanging energy with the grid. Examples of such costs include maintenance costs of the energy storage technology (explained by $\alpha_{t}^{G S}$ and $\alpha_{t}^{S G}$ ), or the maximum amount of power which can be absorbed by the grid (addressed through $\alpha_{t}^{S G}$ and $\alpha_{t}^{W G}$ ). For example, when the grid cannot absorb energy anymore, we have $\alpha_{t}^{S G}=P_{t}^{S G}$ and $\alpha_{t}^{W G}=P_{t}^{W G}$; whence the system owner earns nothing by selling energy to the grid during that time step. In addition to these explicit costs, the system's operation and penetrating power to the grid may impact the real-time electricity price, especially if the storage size and amounts exchanged are significant. This price impact is captured through the transaction cost parameters. One may even consider impacts on the electricity price, and consequently the transaction cost parameters, to be functions of amount exchanged in the same or previous time intervals. However, in this paper, we assume that the transaction cost parameters are constants.

We refer to $C_{t}\left(\tilde{\mathbf{P}}_{t}, X_{t}\right)$ as the stage cost. A negative cost should be interpreted as revenue. The total system operational cost over the planning horizon $[0, T)$ then equals:

$$
\sum_{t=0}^{T_{N}-1} C_{t}\left(\tilde{\mathbf{P}}_{t}, X_{t}\right) .
$$


Describing the uncertain nature of electricity price $\tilde{\mathbf{P}}_{t}$ and time-varying loads and energy supplies require some modeling assumptions, which are explained next. In this paper, similar numerical values are used for prices, i.e., $\tilde{P}_{t}^{G S}=\tilde{P}_{t}^{G D}=\tilde{P}_{t}^{S G}=\tilde{P}_{t}^{W G}=\tilde{P}_{t}$; this is acceptable for most market situations.

[I] Energy Price $\left(\tilde{P}_{t}\right)$ : We assume a reduced form stochastic model accounting for intraday, weekly, and annual seasonality for the electricity spot prices. The deterministic seasonal component of $\tilde{P}_{t}$ is composed of an hour-of-day seasonal factor $P_{t}^{\text {hour }}$, a day-of-week seasonal term $P_{t}^{\text {day }}$, and a monthof-year seasonal part, $P_{t}^{\text {month }}$. These constants $P_{t}^{\text {hour }}, P_{t}^{\text {day }}$, and $P_{t}^{\text {month }}$ are calculated as the average electricity price, respectively, over each of the hours of a day, over each of the days of a week, and over each of the months of a year. Hence, the energy price (in $\$ / \mathrm{MWh}$ ) over the $t^{\text {th }}$ time step is decomposed as

$$
\tilde{P}_{t}=P_{t}^{\text {hour }}+P_{t}^{\text {day }}+P_{t}^{\text {month }}+\tilde{Y}_{t}^{P} .
$$

This model explicitly incorporates price seasonality. The deseasonalized price $\tilde{Y}_{t}^{P}$ has the initial value $\tilde{Y}_{0}^{P}=\tilde{P}_{0}$ $P_{0}^{\text {hour }}-P_{0}^{\text {day }}-P_{0}^{\text {month }}$.

Various reduced-form stochastic processes, as well as structural models, for $\tilde{Y}_{t}^{P}$ have been discussed in the literature. For a list of these processes and their characteristics, the reader is referred to [14]. Mean-reversion and the presence of spikes (or tail fatness) have been addressed as the main features of the electricity spot price distribution. Therefore, to capture these notable properties of energy prices, mean-reverting jump diffusion models have gained popularity, e.g., see [24], [25]. As a result, we also consider a mean-reverting jump diffusion model for $\tilde{Y}_{t}^{P}$ in this paper:

$$
d \tilde{Y}_{t}^{P}=\lambda_{P}\left(\mu_{P}-\tilde{Y}_{t}^{P}\right) d t+\sigma_{P} d \tilde{W}_{t}+\tilde{J}_{t} d \tilde{q}_{t},
$$

assuming that $\tilde{Y}_{t}^{P}$ evolves over continuous time. Here, $\lambda_{P}$ is the speed of mean-reversion, and $\mu_{P}$ and $\sigma_{P}$ denote the mean and volatility, respectively. In equation (6), $d \tilde{W}_{t}$ is the increment of the standard Brownian motion, $\tilde{J}_{t}$ indicates the proportional random jump size, and $d \tilde{q}_{t}$ is a Poisson process with intensity $l$.

[II] Time-varying Energy Demand $\left(D_{t}\right)$ : Similar to electricity prices, loads are modeled through the hour-of-day, dayof-week, and month-of-year seasonal components, denoted respectively by $D_{t}^{\text {hour }}, D_{t}^{\text {day }}$, and $D_{t}^{\text {month }}$. Thus a reasonable approximation for the energy demand (in MWh) over the $t^{\text {th }}$ time step can be

$$
D_{t}=D_{t}^{\text {hour }}+D_{t}^{\text {day }}+D_{t}^{\text {month }}+\mathbb{E}\left[\tilde{Y}_{t}^{D}\right],
$$

where $\tilde{Y}_{t}^{D}$ captures stochastic fluctuations in load. Often in the literature, the deseasonalized load $\tilde{Y}_{t}^{D}$ is addressed by a linear autoregressive model, e.g., see [26], [27],

$$
\tilde{Y}_{t}^{D}=\phi_{D} \tilde{Y}_{t-1}^{D}+\sigma_{D} \sqrt{\Delta t} \tilde{\epsilon}_{t}, \quad \tilde{\epsilon}_{t} \sim \mathcal{N}(0,1)
$$

with the initial value $Y_{0}^{D}=D_{0}-D_{0}^{\text {hour }}-D_{0}^{\text {day }}-D_{0}^{\text {month }}$, and the given constants $\left|\phi_{D}\right|<1$ and $\sigma_{D}$. Therefore, $\mathbb{E}\left[\tilde{Y}_{t}^{D}\right]=$ $\phi_{D}^{t} Y_{0}^{D}$ and, consequently, we have

$$
D_{t}=D_{t}^{\text {hour }}+D_{t}^{\text {day }}+D_{t}^{\text {month }}+\phi_{D}^{t} Y_{0}^{D} .
$$

[III] Time-varying Energy Supply $\left(E_{t}\right)$ : The power delivered by a wind turbine is often represented by its power curve, which establishes a relation between the wind speed and the power. Cubic or approximate cubic power curves have been commonly used to represent the power curves of variable speed wind turbine generators, e.g., see [28], [29]. Here, we consider a power curve model in which the non-linear relationship between power and wind speed is represented by a cubic expression that saturates at the rated power output. Namely, we let the power output $p_{\tilde{W}_{t}}^{(1)}$ (in MW) be given by,

$$
p_{\tilde{W}_{t}}^{(1)}=\left\{\begin{array}{ll}
0 & \text { if } \tilde{W}_{t}<0 \text { or } \tilde{W}_{t}>25 \\
10^{-6} \times \frac{1}{2} A \nu C_{\mathbf{p}} \tilde{W}_{t}^{3} & \text { if } 0 \leq \tilde{W}_{t}<v_{r} \\
p_{r} & \text { if } v_{r} \leq \tilde{W}_{t} \leq 25
\end{array},\right.
$$

where $\tilde{W}_{t}$ denotes the wind speed measured in $\mathrm{m} / \mathrm{s}$ and $v_{r}$ represents the minimal wind speed corresponding to the rated power. In equation (10), $A$ captures the area in $\mathrm{m}^{2}$ swept by the rotor blades of the turbine, $p_{r}$ is the rated power in MW, $C_{\mathbf{p}}$ denotes the power coefficient parameter with the maximum theoretical value of $\frac{16}{27} \approx 0.59$, and $\nu \approx 1.3 \mathrm{~kg} / \mathrm{m}^{3}$ is the density of air, e.g., see [30]. In the sequel, we make the approximation of disregarding the possibility of having wind speed beyond the cut-off speed.

We assume that we have 50 identical wind turbines, each of which has the rated power of $p_{r}=4 \mathrm{MW}$, with the power coefficient $C_{\mathbf{p}}=0.5$ and $A=50^{2} \pi$ (in $\mathrm{m}^{2}$ ). Also, $v_{r} \approx$ $11.62 \mathrm{~m} / \mathrm{s}$. Velocity of the wind is assumed to be equal to $\tilde{W}_{t}=\left(\tilde{Y}_{t}^{E}+\mu_{E}\right)^{2}$, with given $\tilde{W}_{0}$ and $\mu_{E}$. Here, $\tilde{Y}_{t}^{E}$ evolves according to an AR(1) model (e.g., see [31]):

$$
\tilde{Y}_{t}^{E}=\phi_{E} \tilde{Y}_{t-1}^{E}+\sigma_{E} \sqrt{\Delta t} \tilde{\epsilon}_{t}, \quad \tilde{\epsilon}_{t} \sim \mathcal{N}(0,1),
$$

with the deterministic initial value $\tilde{Y}_{0}^{E}=0$ and the given $\left|\phi_{E}\right|<1$ and $\sigma_{E}$. Using the fact that $\left\{\tilde{\epsilon}_{i}\right\}_{i=1}^{t}$ are independent standard normal random variables and the $6^{\text {th }}$ moment formula of standard normals, we can compute $\mathbb{E}\left[\tilde{W}_{t}^{3}\right]$. Thus, the (total) wind energy output (measured in MWh) from the 50 wind turbines over the $t^{\text {th }}$ time step (one hour) can approximately be computed by,

$$
\begin{aligned}
E_{t}= & 50 \times \min \left\{\frac { 5 0 ^ { 2 } \pi \nu } { 2 \times 1 0 ^ { 6 } } \left(\mu_{W}(t)^{6}+15 \mu_{W}(t)^{4} \sigma_{E}^{2}\left(\frac{1-\phi_{E}^{2 t}}{1-\phi_{E}^{2}}\right)\right.\right. \\
& \left.\left.+45 \mu_{W}(t)^{2} \sigma_{E}^{4}\left(\frac{1-\phi_{E}^{2 t}}{1-\phi_{E}^{2}}\right)^{2}+15 \sigma_{E}^{6}\left(\frac{1-\phi_{E}^{2 t}}{1-\phi_{E}^{2}}\right)^{3}\right), 4\right\},
\end{aligned}
$$

where $\mu_{W}(t) \stackrel{\text { def }}{=} \phi_{E}^{t} \tilde{Y}_{0}^{E}+\mu_{E}$.

It is worth mentioning that there are alternative approaches for modeling the wind energy, e.g., see [32]. 


\section{A. Constraints and Admissible Energy Flows}

Given the exogenous parameter values $E_{t}, D_{t}$, and the storage level $S_{t}$ at time step $t$, the following constraints are imposed on the energy flows in (1):

$$
\begin{aligned}
& x_{t}^{W D}=\min \left\{E_{t}, D_{t}\right\}, \\
& x_{t}^{W S}+x_{t}^{W D}+x_{t}^{W G}=E_{t}, \\
& x_{t}^{G D}+\eta^{D} x_{t}^{S D}+x_{t}^{W D}=D_{t}, \\
& x_{t}^{S D}+x_{t}^{S G} \leq \underline{S}_{t} S^{\text {cap }}+\eta^{\mathrm{C}}\left(x_{t}^{G S}+x_{t}^{W S}\right), \\
& \eta^{\mathrm{C}}\left(x_{t}^{G S}+x_{t}^{W S}\right)-\left(x_{t}^{S D}+x_{t}^{S G}\right) \leq \bar{S}_{t} S^{\mathrm{cap}}, \\
& x_{t}^{S D}+x_{t}^{S G} \leq \Delta S^{D} S^{\text {cap }}, \\
& \eta^{\mathrm{C}}\left(x_{t}^{G S}+x_{t}^{W S}\right) \leq \Delta S^{\mathrm{C}} S^{\text {cap }},
\end{aligned}
$$

where the maximum charging rate $0 \leq \Delta S^{\mathrm{C}} \leq 1$ and the maximum discharging rate $0 \leq \Delta S^{D} \leq 1$ are the maximum fraction of the storage which can be charged or discharged, respectively, over the $t^{\text {th }}$ time interval. Here, $\bar{S}_{t}=$ $S^{\max }-\left(1-\gamma_{\Delta t}\right) S_{t}$ and $\underline{S}_{t}=\left(1-\gamma_{\Delta t}\right) S_{t}-S^{\min }$ represent the maximum charging and discharging potential in one time step. The non-negative constants $S^{\min }$ and $S^{\max }$ indicate the minimum and maximum acceptable charge levels, which are percentages of the maximum capacity $S^{\text {cap }}$. These constants, respectively, reflect the maximum depth of discharge due to cycle life considerations and storage degradation over time that precludes reaching to the maximum storage capacity. Constraint (15) implies that the demand is fully satisfied through the grid, the storage, or the wind power generator. Constraint (16) ensures $S_{t+1} \geq S^{\text {min }}$ and constraint (17) guarantees that $S_{t+1} \leq S^{\max }$. Constraints (18) and (19) prevent the storage device from charging or discharging faster than what is allowed.

The model described above permits concurrent charge and discharge of the storage within the same time step. However, routing energy from the grid to the user via the storage might not be efficient, and some literature such as [33] circumvent it. Such a round-trip flow is, however, unlikely to happen at an optimal strategy of our model, as typically $\eta^{\mathrm{C}} \eta^{D}<1$ and transaction cost parameters have reasonable values.

The system (13)-(19) is always feasible, since $\bar{X}_{t}$, whose elements are defined as below, satisfies the set of constraints.

$$
\begin{aligned}
& \bar{x}_{t}^{W D}=F_{t}, \quad \bar{x}_{t}^{G D}=D_{t}-F_{t}, \quad \bar{x}_{t}^{S D}=0, \\
& \bar{x}_{t}^{W G}=E_{t}-F_{t}, \quad \bar{x}_{t}^{W S}=0, \quad \bar{x}_{t}^{G S}=0, \quad \bar{x}_{t}^{S G}=0,
\end{aligned}
$$

where $F_{t} \stackrel{\text { def }}{=} \min \left\{E_{t}, D_{t}\right\}$. This strategy is the unique feasible strategy to serve the demand $D_{t}$, in the absence of a storage device, i.e., $S^{\text {cap }}=0 \mathrm{MWh}$.

From the equality constraints (13)-(15), the three decision variables $x_{t}^{W D}, x_{t}^{W S}$, and $x_{t}^{S D}$ can be fully determined using the other four decision variables. Denote

$$
x_{t} \stackrel{\text { def }}{=}\left(x_{t}^{G S}, x_{t}^{G D}, x_{t}^{S G}, x_{t}^{W G}\right)^{\top} .
$$

Therefore, a feasible $x_{t}$ can be explained using constraints (16)-(19), stated in terms of $x_{t}$ in (21), and the two additional inequality constraints

$$
\begin{aligned}
& E_{t}-\min \left\{E_{t}, D_{t}\right\} \geq x_{t}^{W G}, \\
& D_{t}-\min \left\{E_{t}, D_{t}\right\} \geq x_{t}^{G D},
\end{aligned}
$$

to ensure $x_{t}^{W S} \geq 0$ and $x_{t}^{S D} \geq 0$. These constraints together imply that at every time step $t, x_{t}^{G D}=0$ or $x_{t}^{W G}=0$.

Given $E_{t}, D_{t}$, and $S_{t}$, the constraints (16)-(19) and (22)(23) can be presented as a system of linear inequalities $A x_{t} \leq$ $b_{t}$. Throughout this paper, we refer to the set of $x_{t} \in \mathbb{R}_{+}^{4}$ satisfying $A x_{t} \leq b_{t}$ by $\mathcal{X}_{t}$, which is bounded and convex.

\section{B. Myopic and Optimal Risk Neutral Policies}

At any time step $t$, a naive approach to adopt a system operation strategy is to simply minimize the $t^{\text {th }}$ stage cost and solve the following linear programming problem:

$$
\min _{x_{t} \in \mathcal{X}_{t}} C_{t}\left(\tilde{P}_{t}, x_{t}\right) .
$$

This policy, referred to as the myopic policy and denoted henceforth by Policy $_{M}$, discharges the storage as quickly as possible. While computing the myopic policy is straightforward, it entirely ignores the influence of decisions on the future state of the storage charge level, and hence the total operational cost. Hence, it is not a practically interesting strategy.

An alternative to the myopic policy, in which the total cost over the planning horizon is taken into account, is a solution of the following problem

$$
\min _{x_{0} \in \mathcal{X}_{0}, \cdots, x_{T_{N}-1} \in \mathcal{X}_{T_{N}-1}} \mathbb{E}\left[\sum_{t=0}^{T_{N}-1} C_{t}\left(\tilde{P}_{t}, x_{t}\right)\right],
$$

which is referred to as an optimal risk neutral strategy and is denoted by Policy $_{E}$. When the transaction cost parameters are constants (i.e., do not depend on $x_{t}$ ), and parameters $D_{t}$ and $E_{t}$ can be approximated well by some deterministic (but time-varying) values, problem (25) is reduced to a linear programming problem.

Similar to the myopic policy, a solution for problem (25) can be computed easily either through a closed-form formula (for special cases) or using linear programming solution techniques and solvers.

Whether constraints are treated deterministically or probabilistically, Policy ${ }_{E}$ does not depend on the assumed stochastic dynamics model for $\tilde{Y}_{t}^{P}$ and, consequently, for the price $\tilde{P}_{t}$. However, the energy price, appearing in the objective function, is known to be highly volatile and spiky, e.g., see [14]. This fat tail behavior of the energy prices motivates the importance of using some downside risk (tail risk) measure, when looking for an optimal storage operation management strategy.

\section{Risk Analysis AND DOWNSIDE Risk MEASURES}

The price risk is one of the most significant risks in the energy industry [16]. One of the most widely used risk measures is Value-at-Risk (VaR), e.g., see [16] or [34]. This downside risk measure is particularly appropriate for loss distributions with fat tail behavior such as that in the energy prices. For a given time horizon and confidence level $\beta$, the 


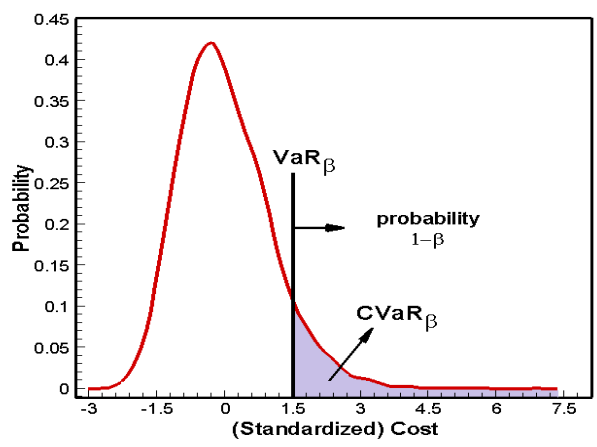

Fig. 2: Probability distribution function of operational cost.

value-at-risk at the confidence level $\beta$ is the smallest cost (loss in market value) over the time horizon that is exceeded with probability (no greater than) $1-\beta$, e.g., see [19]. In other words, $\operatorname{VaR}_{\beta}$ (cost) is the level $\beta$-quantile of the loss distribution, i.e.,

$$
\operatorname{VaR}_{\beta}(\text { cost })=\inf \{\ell \in \mathbb{R}: \quad \operatorname{Pr}(\cos t \leq \ell) \geq \beta\} .
$$

Figure 2 illustrates the probability distribution function of the total system operational cost, using the input data presented in Section V and Policy ${ }_{E}$. Here we assumed that all of the transaction cost parameters are equal to 0 . Table I reports the VaR values of the total costs corresponding to Policy ${ }_{E}$ and Policy $_{M}$. As the results in this table indicate, while by minimizing the expected operational cost and adopting Policy ${ }_{E}$, one expects to incur an operational cost of $\$ 15,458,318.42$, for example by looking at $\mathrm{VaR}_{90 \%}$, in $10 \%$ of cases the incurred cost will be $\$ 4,542,409.45$ (around 29.38\%) more than what is expected. Thus, to obtain an operation plan with less exposure to market risk and variations in the electricity spot price, it is important to include a downside risk measure in the objective function.

While VaR is still a firm-wide measure of risk in calculating regulatory and economic capital, the VaR function is nonconvex, non-smooth, and has many local minima, see e.g., [19]. An attractive alternative to $\mathrm{VaR}$ is the coherent risk measure Conditional Value-at-Risk (CVaR), also known as average Value-at-Risk, mean excess loss, or mean shortfall.

For a given time horizon $\bar{t}$ and confidence level $\beta, \mathrm{CVaR}_{\beta}$ is the conditional expectation of the loss above $\mathrm{VaR}_{\beta}$ for the time horizon $\bar{t}$. Thus, $\mathrm{CVaR}_{\beta}$ can be defined as

$$
\mathrm{CVaR}_{\beta}(\cos \mathrm{t})=\mathbb{E}\left[\operatorname{cost}: \quad \cos \mathrm{t} \geq \operatorname{VaR}_{\beta}(\cos \mathrm{t})\right] .
$$

Without referencing to $\mathrm{VaR}$, a more direct way of defining CVaR is, see, e.g., [35]:

$$
\operatorname{CVaR}_{\beta}(\cos t)=\min _{\alpha}\left(\alpha+\frac{1}{1-\beta} \mathbb{E}\left[[\cos t-\alpha]^{+}\right]\right),
$$

where $[z]^{+}=\max (z, 0)$. Minimizing CVaR typically leads to a strategy with a small VaR. Next, we discuss the CVaR risk consideration for the system operational cost of interest.
TABLE I: $\operatorname{VaR}_{\beta}$ of the system operational cost corresponding to Policy ${ }_{E}$ and Policy $_{M}$ over 168 hours computed using 20,000 Monte Carlo paths. Expected values of the total cost corresponding to Policy ${ }_{E}$ and Policy ${ }_{M}$ equal $\$ 15,458,318.42$ and $\$ 15,530,033.15$, respectively.

\begin{tabular}{|c|c|c|c|}
\multicolumn{4}{|c|}{ Policy $_{E}$} \\
\hline$\beta[\%]$ & $\operatorname{VaR}_{\beta}[\$]$ & $\begin{array}{c}\text { Absolute } \\
\text { Difference [\$] }\end{array}$ & $\begin{array}{c}\text { Relative } \\
\text { Difference [\%] }\end{array}$ \\
\hline \hline 75 & $17,659,736.25$ & $2,201,417.83$ & 14.24 \\
80 & $18,241,611.01$ & $2,783,292.59$ & 18.01 \\
85 & $19,049,807.51$ & $3,591,489.10$ & 23.23 \\
90 & $20,000,727.86$ & $4,542,409.45$ & 29.38 \\
95 & $21,877,231.73$ & $6,418,913.31$ & 41.52 \\
99 & $25,336,499.63$ & $9,878,181.21$ & 63.90 \\
99.90 & $30,041,183.60$ & $14,582,865.19$ & 94.34 \\
\hline
\end{tabular}

\begin{tabular}{|c|c|c|c|}
\multicolumn{4}{|c|}{ Policy $_{M}$} \\
\hline$\beta[\%]$ & $\operatorname{VaR}_{\beta}[\$]$ & $\begin{array}{c}\text { Absolute } \\
\text { Difference [\$] }\end{array}$ & $\begin{array}{c}\text { Relative } \\
\text { Difference [\%] }\end{array}$ \\
\hline \hline 75 & $17,715,420.01$ & $2,185,386.86$ & 14.07 \\
80 & $18,291,168.95$ & $2,761,135.81$ & 17.78 \\
85 & $19,100,810.54$ & $3,570,777.39$ & 22.99 \\
90 & $20,137,750.95$ & $4,607,717.80$ & 29.67 \\
95 & $21,815,224.10$ & $6,285,190.96$ & 40.47 \\
99 & $25,496,654.67$ & $9,966,621.52$ & 64.18 \\
99.90 & $30,118,873.21$ & $14,588,840.06$ & 93.94 \\
\hline
\end{tabular}

\section{Optimal Risk Averse Energy Storage Policy}

An optimal risk averse (deterministic) policy can be derived by solving the following problem:

$$
\begin{array}{ll}
\min & \omega_{\mathbb{E}} \mathbb{E}\left[\sum_{t=0}^{T_{N}-1} C_{t}\left(\tilde{P}_{t}, x_{t}\right)\right]+\omega_{\rho} \rho\left[\sum_{t=0}^{T_{N}-1} C_{t}\left(\tilde{P}_{t}, x_{t}\right)\right], \\
\text { s.t. } & x_{0} \in \mathcal{X}_{0}, \cdots, x_{T_{N}-1} \in \mathcal{X}_{T_{N}-1} .
\end{array}
$$

Here, $\rho[\cdot]$ is a risk measure. The non-negative parameters $\omega_{\mathbb{E}}$ and $\omega_{\rho}$, where $\omega_{\mathbb{E}}+\omega_{\rho}=1$, capture the aversion of the decision maker to the price risk. We focus on the conditional value-at-risk as the risk measure. Using equation (28), a mean-CVaR optimal deterministic energy storage policy is determined by:

$$
\begin{aligned}
& \min _{x \in \mathbb{R}_{+}^{4 T}} \omega_{\mathbb{E}} \mathbb{E}\left[\tilde{c}^{\top} x\right]+\omega_{\rho} \mathrm{CVaR}_{\beta}\left[\tilde{c}^{\top} x\right] \\
= & \min _{\alpha \in \mathbb{R}, x \in \mathbb{R}_{+}^{4 T}} \omega_{\mathbb{E}} \mathbb{E}\left[\tilde{c}^{\top} x\right]+\omega_{\rho} \alpha+\frac{\omega_{\rho}}{1-\beta} \mathbb{E}\left[\left[\tilde{c}^{\top} x-\alpha\right]^{+}\right],
\end{aligned}
$$

where $x=\left(x_{0}, \cdots, x_{T_{N}-1}\right)^{\top}$ and $\tilde{c}=\left(\tilde{c}_{0}, \cdots, \tilde{c}_{T_{N}-1}\right)^{\top}$. To solve problem (30), [35] suggest to replace the piecewise linear function $[z]^{+}$with a set of linear constraints, as below,

$$
\begin{array}{cl}
\min _{\alpha \in \mathbb{R}, x \in \prod_{t=0}^{T_{N}-1} \mathcal{X}_{t}} & \omega_{\mathbb{E}} \mathbb{E}\left[\tilde{c}^{\top} x\right]+\omega_{\rho} \alpha+\frac{\omega_{\rho}}{M(1-\beta)} \sum_{\ell=1}^{M} z_{\ell} \\
& z_{\ell}-x^{\top} \tilde{c}^{(\ell)}+\alpha \geq 0, \quad \ell=1, \cdots, M, \\
& z_{\ell} \geq 0, \quad \ell=1, \cdots, M .
\end{array}
$$

Here, $M$ denotes the number of Monte Carlo scenario paths. Here, the Monte Carlo simulation is used to approximate the 
mathematical expectations (for mean as well as the expected value in the definition of (VaR) in the objective function.

This approach can quickly become computationally rather expensive and inefficient as the number of simulation paths increases; each new scenario path introduces a new decision variable $z_{\ell}$ and a new constraint to the optimization problem. However, to have an accurate estimation of the tail of the cost distribution, a large number of Monte Carlo paths is required.

Alternatively, using the smoothing technique in [36], the function $[z]^{+}$can be approximated with a continuously differentiable piecewise quadratic function $\varrho_{\epsilon}(z)$ using a small resolution parameter $\epsilon>0$ :

$$
\varrho_{\epsilon}(z)= \begin{cases}z & \text { if } z>\epsilon \\ \frac{z^{2}}{4 \epsilon}+\frac{1}{2} z+\frac{1}{4} \epsilon & \text { if }-\epsilon \leq z \leq \epsilon \\ 0 & \text { if } z<-\epsilon\end{cases}
$$

Note that $\varrho_{\epsilon}(z) \geq 0$ for every $z$. It is shown in [36] that this smoothing approach can be significantly more efficient than the linear programming formulation (31) for the CVaR minimization problem. The convergence of stationary points of the smoothed sample average approximation problem, with the increase of sample size, has been investigated in [37]. More precisely, [37] proves that accumulation points of the stationary points of the smoothed sample average approximation problem, to solve the $\varrho_{\epsilon}$-smoothed problem, are almost surely weak stationary points of their counterparts in the true problem, as the sample size increases.

Applying function (32), the objective function in (30) is then reduced to the following convex and continuously differentiable piecewise quadratic function:

$$
\begin{aligned}
& \omega_{\mathbb{E}} \mathbb{E}\left[\tilde{c}^{\top} x\right]+\omega_{\rho} \alpha+\frac{\omega_{\rho}}{1-\beta} \mathbb{E}\left[\varrho_{\epsilon}\left(\tilde{c}^{\top} x-\alpha\right)\right] \\
& =\omega_{\mathbb{E}} \mathbb{E}[\tilde{c}]^{\top} x+\omega_{\rho} \alpha+\frac{\omega_{\rho}}{M(1-\beta)} \sum_{\ell=1}^{M} \varrho_{\epsilon}\left(x^{\top} \tilde{c}^{(\ell)}-\alpha\right) .
\end{aligned}
$$

We refer to a solution of problem (29) with an objective function as in (33) by Policy ${ }_{C}$. In this formulation, the number of decision variables or the constraints do not depend on the number of Monte Carlo sample paths. This problem can be solved using an augmented Lagrangian method, e.g., see Section 17.3 of [38]. The augmented Lagrangian, denoted by $\mathcal{L}_{A}(x, s, \alpha, \lambda ; \mu)$, for this problem can be written as below:

$$
\begin{aligned}
& \omega_{\mathbb{E}} \mathbb{E}[\tilde{c}]^{\top} x+\omega_{\rho} \alpha+\frac{\omega_{\rho}}{M(1-\beta)} \sum_{\ell=1}^{M} \varrho_{\epsilon}\left(x^{\top} \tilde{c}^{(\ell)}-\alpha\right) \\
& -\sum_{t=0}^{T_{N}-1} \lambda_{t}^{\top}\left(A x_{t}-b_{t}+s_{t}\right)+\frac{\mu}{2} \sum_{t=0}^{T_{N}-1}\left\|A x_{t}-b_{t}+s_{t}\right\|_{2}^{2},
\end{aligned}
$$

where $x_{t} \geq 0$ and the slack variables $s_{t} \geq 0$. The vector $\lambda_{t}$ includes the Lagrange multipliers corresponding to the constraints $A x_{t}+s_{t}=b_{t}$. At every iteration $k$, the algorithm then fixes the penalty parameter $\mu^{(k)}>0$, fixes the Lagrange multipliers at the current estimate $\lambda^{(k)}$, and performs minimization of the augmented Lagrangian with respect to $x, s$, and $\alpha$. The computed approximate minimizer is then used to update the penalty parameter and Lagrange multipliers. Such



Fig. 3: Load over the time horizon (168 hours).

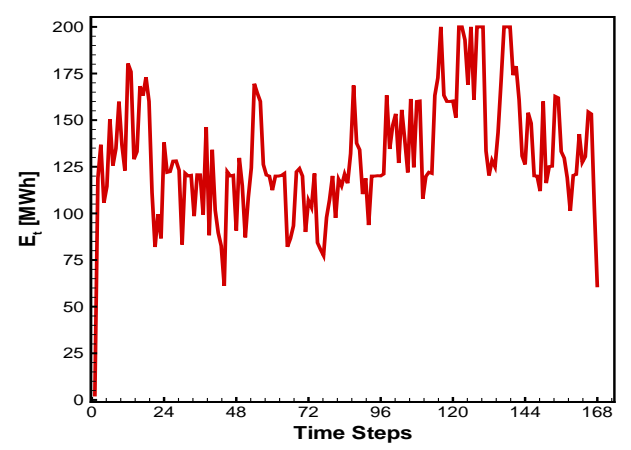

Fig. 4: Wind energy (average of 50 simulated paths).

a minimizer can be calculated using an interior point method [39] for nonlinear minimization with bound constraints.

\section{Numerical ILLUSTRATION}

We use five-minute prices (in $\$ / \mathrm{MWh}$ ) and hourly demands (in MW) for NYISO from 12 am of January 1, 2007 (Monday) to $12 \mathrm{am}$ of December 31, 2011 (Saturday). These data sets are publicly available at NYISO website. We replace the negative prices with $\$ 1 / \mathrm{MWh}$, and take the average of 12 measurements from five-minute prices for each hour to derive hourly prices. The estimation process for the deterministic seasonal trend is then as follows: (1) For every hour of a day, $P_{t}^{\text {hour }}$ is set as the mean of the price of that hour over all of the days in our data set. Then for every hour, the computed $P_{t}^{\text {hour }}$ is subtracted from the hourly prices to derive hourly residual prices. (2) For each day in a week, $P_{t}^{\text {day }}$ is computed as the mean of the average hourly residual prices per day over all of the weeks in the historical data set. Then for each hour this value is subtracted from the hourly residual prices to obtain updated hourly residual prices. (3) For each month of a year, $P_{t}^{\text {month }}$ is the mean of the average updated hourly residual prices per month over the five years 2007 to 2011. This value is then subtracted from each updated hourly residual price to attain deseasonalized hourly prices. A similar process is carried out for loads. The estimated values for seasonal terms are presented in Tables II, III, and IV.

Using the maximum likelihood method, we then obtain estimations for the parameters in $\tilde{Y}_{t}^{P}$ and $\tilde{Y}_{t}^{D}$. This is reported 
TABLE II: Hour of Day Seasonality Factors

\begin{tabular}{|c|c|c|c|}
\hline Hour & Time Interval & Demand $D_{t}^{\text {hour }}[\mathbf{M W}]$ & Price $P_{t}^{\text {hour }}[\$ / \mathbf{M W h}]$ \\
\hline \hline 0 & {$[12 \mathrm{am}, 1 \mathrm{am})$} & 5159.62 & 52.92 \\
\hline 1 & {$[1 \mathrm{am}, 2 \mathrm{am})$} & 4943.20 & 47.81 \\
\hline 2 & {$[2 \mathrm{am}, 3 \mathrm{am})$} & 4819.20 & 43.88 \\
\hline 3 & {$[3 \mathrm{am}, 4 \mathrm{am})$} & 4781.16 & 42.30 \\
\hline 4 & {$[4 \mathrm{am}, 5 \mathrm{am})$} & 4885.42 & 44.07 \\
\hline 5 & {$[5 \mathrm{am}, 6 \mathrm{am})$} & 5225.77 & 48.49 \\
\hline 6 & {$[6 \mathrm{am}, 7 \mathrm{am})$} & 5713.78 & 57.95 \\
\hline 7 & {$[7 \mathrm{am}, 8 \mathrm{am})$} & 6160.71 & 61.54 \\
\hline 8 & {$[8 \mathrm{am}, 9 \mathrm{am})$} & 6515.21 & 66.08 \\
\hline 9 & {$[9 \mathrm{am}, 10 \mathrm{am})$} & 6756.23 & 71.13 \\
\hline 10 & {$[10 \mathrm{am}, 11 \mathrm{am})$} & 6898.87 & 72.75 \\
\hline 11 & {$[11 \mathrm{am}, 12 \mathrm{pm})$} & 6973.70 & 73.00 \\
\hline 12 & {$[12 \mathrm{pm}, 1 \mathrm{pm})$} & 7006.82 & 75.05 \\
\hline 13 & {$[1 \mathrm{pm}, 2 \mathrm{pm})$} & 7015.28 & 77.30 \\
\hline 14 & {$[2 \mathrm{pm}, 3 \mathrm{pm})$} & 7017.51 & 80.85 \\
\hline 15 & {$[3 \mathrm{pm}, 4 \mathrm{pm})$} & 7029.42 & 81.30 \\
\hline 16 & {$[4 \mathrm{pm}, 5 \mathrm{pm})$} & 7036.35 & 85.72 \\
\hline 17 & {$[5 \mathrm{pm}, 6 \mathrm{pm})$} & 6974.70 & 88.22 \\
\hline 18 & {$[6 \mathrm{pm}, 7 \mathrm{pm})$} & 6881.89 & 82.30 \\
\hline 19 & {$[7 \mathrm{pm}, 8 \mathrm{pm})$} & 6773.92 & 80.02 \\
\hline 20 & {$[8 \mathrm{pm}, 9 \mathrm{pm})$} & 6595.16 & 77.30 \\
\hline 21 & {$[9 \mathrm{pm}, 10 \mathrm{pm})$} & 6309.24 & 68.12 \\
\hline 22 & {$[10 \mathrm{pm}, 11 \mathrm{pm})$} & 5918.03 & 61.52 \\
\hline 23 & {$[11 \mathrm{pm}, 12 \mathrm{am})$} & 5492.11 & 56.51 \\
\hline & & & \\
\hline
\end{tabular}

TABLE III: Day of Week Seasonality Factors

\begin{tabular}{|c|c|c|}
\hline Day & Demand $D_{t}^{\text {day }}[\mathbf{M W}]$ & Price $P_{t}^{\text {day }}[\$ / \mathbf{M W h}]$ \\
\hline \hline Monday & 174.19 & 2.43 \\
\hline Tuesday & 266.76 & 2.49 \\
\hline Wednesday & 263.84 & 3.42 \\
\hline Thursday & 224.28 & 1.17 \\
\hline Friday & 146.66 & 0.34 \\
\hline Saturday & -468.64 & -3.65 \\
\hline Sunday & -592.24 & -5.45 \\
\hline
\end{tabular}

TABLE IV: Month of Year Seasonality Factors

\begin{tabular}{|c|c|c|}
\hline Month & Demand $D_{t}^{\text {month }}[\mathbf{M W}]$ & Price $P_{t}^{\text {month }}[\$ / \mathbf{M W h}]$ \\
\hline \hline January & -221.78 & 10.29 \\
\hline February & -253.70 & 6.04 \\
\hline March & -520.57 & -2.71 \\
\hline April & -682.52 & -0.97 \\
\hline May & -454.68 & 1.69 \\
\hline June & 659.15 & 10.29 \\
\hline July & 1454.98 & 13.99 \\
\hline August & 1147.22 & -0.79 \\
\hline September & 297.15 & -8.56 \\
\hline October & -524.26 & -14.23 \\
\hline November & -627.55 & -15.38 \\
\hline December & -307.61 & 0.23 \\
\hline
\end{tabular}

TABLE V: Parameters for $\tilde{Y}_{t}^{P}, \tilde{Y}_{t}^{D}$, and $E_{t}$ \begin{tabular}{|c|c|c|}
\hline$\mu_{P}=4.35$ & $\lambda_{P}=37.48$ & $\sigma_{P}=2.08$ \\
\hline$\phi_{D}=0.97$ & $\sigma_{D}=138.08$ & \\
\hline$\phi_{E}=0.95$ & $\sigma_{E}=0.9$ & $\mu_{E}=3$ \\
\hline
\end{tabular}

TABLE VI: Parameters for Energy Storage

\begin{tabular}{|c|c|c|}
\hline$S^{\text {min }}=0.1$ & $\Delta S^{\mathrm{C}}=0.2$ & $\eta^{\mathrm{C}}=0.75$ \\
\hline$S^{\mathrm{max}}=0.9$ & $\Delta S^{D}=0.25$ & $\eta^{D}=0.9$ \\
\hline$S_{0}=S^{\text {min }}$ & $S^{\text {cap }}=1,000[\mathrm{MWh}]$ & $\gamma_{\Delta t}=0$ \\
\hline
\end{tabular}

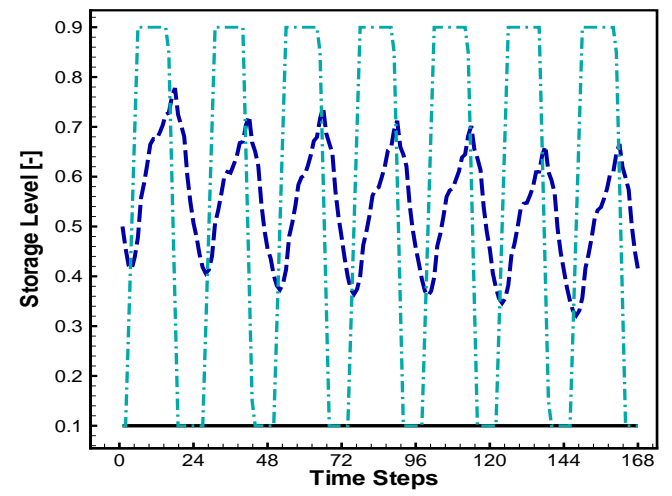

Fig. 5: Storage levels corresponding to Policy ${ }_{E}$ (dotdashed blue line) and Policy ${ }_{M}$ (solid black line). The dashed blue line indicates the expected price (scaled by $\frac{1}{2 P_{0}}$ ).

in Table V, with $l=0.27$, and $\tilde{J}_{t} \sim \mathcal{N}(0.03,0.41)$ for $\tilde{Y}_{t}^{P}$. We let $\tilde{Y}_{0}^{P}=-5.88$ and $\tilde{Y}_{0}^{D}=-63.63$, which are the deseasonal demand and price at hour 12 am of January 1, 2007. In the first week of January 2007, the maximum load and minimum load had been 7,003.60 MW and 4,223.10 MW, respectively. We assume that the energy demand to be served by the system owner is $25 \%$ of the demand calibrated from historical data from NYISO. Demand over the time horizon of our study is illustrated in Fig. 3.

Furthermore, in our numerical studies, to approximate $E_{t}$ we assume $\tilde{Y}_{0}^{E}=0$, and $\phi_{E}, \sigma_{E}, \mu_{E}$ as in Table V. In addition, for the energy storage we use the values in Table VI. We then use the average of 50 path samples as $E_{t}$ in our case study. The energy $E_{t}$ over the time horizon 168 hours is illustrated in Fig. 4. Next, in V-A, different policies are compared. Sensitivity to the transaction cost parameters is investigated in V-B.

\section{A. Policy Analysis}

Figure 5 illustrates storage levels corresponding to Policy and Policy ${ }_{M}$, when all of the transaction cost parameters are equal to zero. The dashed line in this plot indicates the scaled expected price. Policy ${ }_{M}$ discharges the storage to the minimum level $S^{\min } S^{\text {cap }}$ as fast as possible (in this example, in the first time step) and keeps it at the minimum level for the rest of the planning horizon. In contrast, Policy ${ }_{E}$ increases the storage level toward the maximum level $S^{\max } S^{\text {cap }}$ when the gradient of the expected price is positive, but discharges it toward the minimum level $S^{\mathrm{min}} S^{\text {cap }}$ when the expected price is decreasing. The speed of switching between $S^{\min }$ and $S^{\max }$ depends on $\Delta S^{\mathrm{C}}$ and $\Delta S^{\mathrm{D}}$. For example, when $\Delta S^{\mathrm{C}} \geq 0.8$ and $S^{\mathrm{D}} \geq 0$, Policy ${ }_{E}$ is a two-threshold policy with abrupt changes in $S_{t}$ and moves with the gradient of the expected price, when all of the transaction cost parameters are equal to zero. For $\Delta S^{\mathrm{C}}=0.20$ and $\Delta S^{\mathrm{D}}=0.25$, as assumed in Table VI, switching between the maximum and minimum charge levels requires four time steps (hours). Note that the configurations of both Policy ${ }_{E}$ and Policy ${ }_{M}$ depend on neither 


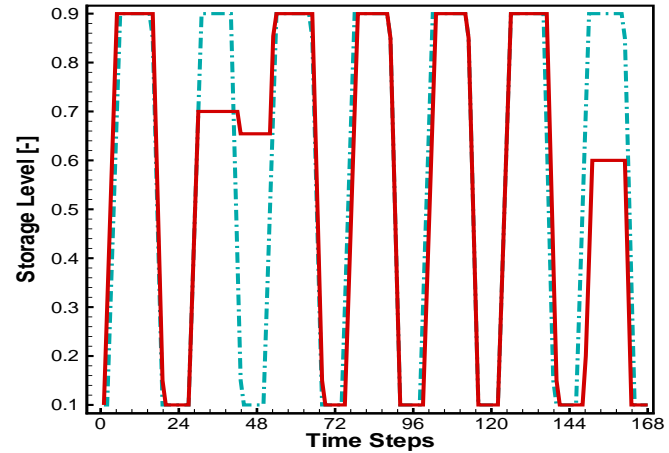

(a) $\beta=85 \%$

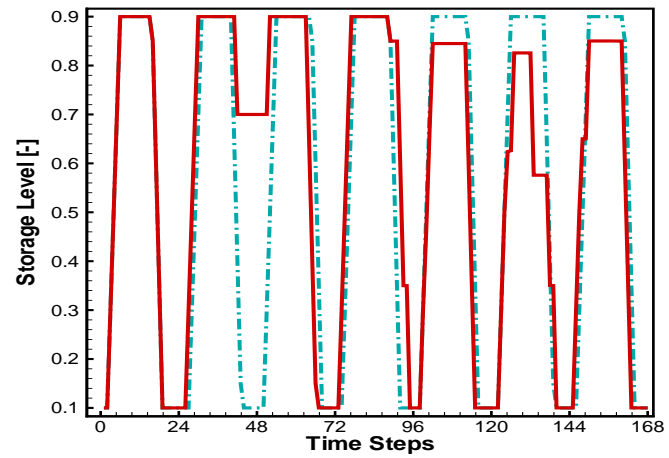

(c) $\beta=99.00 \%$



(b) $\beta=90 \%$

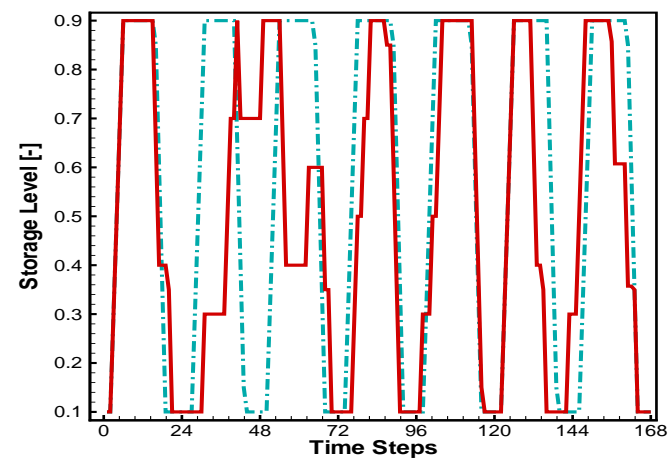

(d) $\beta=99.90 \%$

Fig. 6: Storage levels corresponding to Policy ${ }_{C}$ (solid red line) and Policy ${ }_{E}$ (dotdashed blue line). In these plots $\omega_{\mathbb{E}}=\frac{1}{51}$ and $\omega_{\rho}=\frac{50}{51}$ and the transaction cost parameters equal zero.

the stochastic fluctuations of $\tilde{Y}_{t}^{P}$ nor the average of spikes in the set of generated scenario paths.

Figure 6 depicts the storage levels of Policy ${ }_{C}$ for several confidence levels $\beta$, when $\omega_{\mathbb{E}}=\frac{1}{51}$ and $\omega_{\rho}=\frac{50}{51}$. Unlike Policy $_{E}$, Policy $_{C}$ is not a two-threshold policy, even when all of the transaction cost parameters are zero; by implementing Policy $_{C}, S_{t}$ may remain in other (interior) storage levels. However, this pattern depends highly on the chosen confidence level $\beta$. As the value of the confidence level increases, the storage profile deviates more from the two-threshold configuration.

The costs and risks of the three policies Policy ${ }_{C}$, Policy ${ }_{E}$, and Policy ${ }_{M}$ are presented in Table VII. Here, $\varepsilon_{\mathrm{CVaR}_{\beta}}$ and $\varepsilon_{\text {mean }}$ denote the relative difference between the CVaR and expected costs of policies, respectively. As the table indicates, by implementing Policy ${ }_{C}$ the incurred operational cost is only about $1 \%$ more than the optimal expected cost. However, by adopting Policy $_{E}$ the risk to contend with could be up to $13 \%$ more than what Policy $_{C}$ would have yielded.

\section{B. Sensitivity to Transaction Cost Parameters}

In this section, we consider nonzero transaction cost parameters and investigate impacts of changes in the transaction cost parameters on the strategies Policy ${ }_{E}$ and Policy ${ }_{C}$.
TABLE VII: Expected value and $\mathrm{CVaR}_{\beta}$ of the operational cost.

\begin{tabular}{|c||c|c|c|}
\hline & Policy $_{C}$ & Policy $_{E}$ & Policy $_{M}$ \\
\hline \hline Mean & $\omega_{E}=0, \omega_{\rho}=1$ & & \\
$\varepsilon_{\text {mean }}$ & $15,483,053.51$ & $15,458,318.42$ & $15,530,033.15$ \\
$\mathrm{CVaR}_{85 \%}$ & $0.16 \%$ & - & $0.46 \%$ \\
$\varepsilon_{\mathrm{CVaR}_{85 \%}}$ & $21,216,461.07$ & $21,529,402.82$ & $21,576,631.39$ \\
\hline Mean & $15,522,149.73$ & $15,458,318.42$ & $15,530,033.15$ \\
$\varepsilon_{\text {mean }}$ & $0.41 \%$ & - & $0.46 \%$ \\
$\mathrm{CVaR}_{90 \%}$ & $21,521,120.95$ & $22,546,664.41$ & $22,593,562.81$ \\
$\varepsilon_{\mathrm{CVaR}}$ & - & $4.77 \%$ & $4.98 \%$ \\
\hline Mean $_{9}$ & $15,591,503.39$ & $15,458,318.42$ & $15,530,033.15$ \\
$\varepsilon_{\text {mean }}$ & $0.86 \%$ & - & $0.46 \%$ \\
$\mathrm{CVaR}_{95 \%}$ & $23,782,833.07$ & $26,158,851.56$ & $26,290,658.08$ \\
$\varepsilon_{\mathrm{CVaR}_{95 \%}}$ & - & $9.99 \%$ & $10.54 \%$ \\
\hline Mean $_{\varepsilon_{\text {mean }}}$ & $15,625,009.39$ & $15,458,318.42$ & $15,530,033.15$ \\
$\mathrm{CVaR}_{99.90 \%}$ & $30,946,785.70$ & $35,127,905.09$ & $35,288,053.30$ \\
$\varepsilon_{\mathrm{CVaR}_{99.90 \%}}$ & - & $13.51 \%$ & $14.02 \%$ \\
\hline
\end{tabular}

Figure 7 illustrates the storage levels associated with the optimal storage policies for three instances of these parameters, when $\beta=90 \%, \omega_{\mathbb{E}}=\frac{1}{51}$, and $\omega_{\rho}=\frac{50}{51}$. A comparison 
of these plots with the plot (b) in Fig. 6 clearly suggests that the transaction cost parameters directly influence the structure of the optimal storage policy and its storage level. In Fig. 7 (a), the profile of the storage corresponding to Policy $E$ is exactly the same of the storage level of Policy ${ }_{E}$ in Fig 6(b), which had been obtained under the assumption that all of the transaction cost parameters are equal to zero. However, even for this small change in the transaction cost parameters, Policy $_{C}$ yields a different storage profile within the time steps $64-67$ and $123-140$, and $151-162$. As $\alpha^{G S}$ increases,

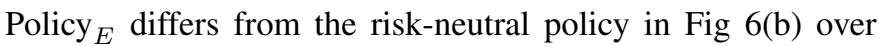
the time steps $31-43$, in which the storage will not be charged as it would have been previously. It makes sense since now buying energy from the grid to store in the storage device for next time steps incurs additional cost due to the transaction cost $\alpha^{G S}>0$, and would no longer be beneficial. As this transaction cost increases, for example to $\alpha^{G S}=6$, as in Fig. 7 (c), the storage level further decreases during the time steps $3-16,28-43,75-91,99-115$, and $123-139$. By increasing the transaction cost parameter $\alpha^{G S}$, the storage level corresponding to Policy ${ }_{C}$ is further pushed toward the interior levels. This analysis suggests the importance of including the transaction cost parameters and their estimation in the energy storage management problem, particularly when a risk averse policy is sought and the objective function includes the $\mathrm{CVaR}$ risk measure with a positive risk aversion parameter $\omega_{\rho}$. A thorough analysis on the impact of these parameters, when the objective function and constraints are linear, can be derived using the results from parametric linear programming (e.g., see [40]).

\section{CONClusions And Future Work}

An optimal energy storage management problem under the headings of risk consideration and transaction costs of exchanging energy with an electrical grid was studied in this paper. A renewable power generation source and energy storage are managed to fully satisfy an energy load with the capability to trade energy with the grid. The fat tail behavior of energy prices and a risk analysis of the optimal risk neutral strategy were provided to motivate downside risk consideration in the problem. An efficient method to compute mean-CVaR optimal strategies was developed based on a smoothing piecewise quadratic function and an augmented Lagrangian method. Using the real world data from NYISO, we showed that the optimal risk aversion strategy can be quite different from the optimal risk neutral strategy. This difference becomes more prominent as the CVaR confidence level $\beta$ increases. A sensitivity analysis of the optimal storage policies, their expected costs, and price risks was provided.

Several issues are left for future investigation. Extending this study for more general storage models, e.g., considering the impact of full charges or discharges on the storage life or constraints regarding safety concerns or time varying efficiency parameters, is an interesting direction for future work. Extending and applying the developed methodology and risk analysis for more realistic settings, e.g., other applications or other distributional model assumptions for the uncertain



(a) $\alpha^{G S}=1$



(b) $\alpha^{G S}=3$



(c) $\alpha^{G S}=6$

Fig. 7: Storage levels corresponding to Policy ${ }_{C}$ (solid red line) and Policy ${ }_{E}$ (dotdashed green line) with three non-zero values for $\alpha^{G S}$. Other transaction cost parameters are equal to zero.

demands and particularly energy prices would also be a beneficial study. Furthermore, when reasonable values for loads and energy supplies are not available, the constraints determining admissible energy flows can be replaced with their corresponding chance constraints. Investigating the structure of the optimal risk averse and risk neutral policies with chance constraints to define admissible policies over the planning horizon would be insightful. Solution techniques such as [41] can then be adopted. Including capital cost and opportunity cost in the total operational cost within the risk averse operation 
management framework can be another research avenue.

For managing the operation of an energy storage, one may adopt a robust optimization approach with an appropriate uncertainty set, e.g., see [42], [43] for the latest research on the theory of robust optimization, and [44], [45] for applications of this approach for planning purposes in energy and power systems. It is interesting to compare the configuration and performance of the robust risk-averse energy storage operation management policy with those of the nominal risk-averse policy.

\section{ACKNOWLEDGMENT}

The authors would like to thank the editor and reviewers for their sincere comments on improving the quality of this paper.

\section{REFERENCES}

[1] P. F. Ribeiro, B. K. Johnson, M. L. Crow, A. Asroy, and Y. Liu, "Energy storage systems for advanced power applications," Proceedings of the IEEE, vol. 89, no. 12 , pp. 1744-1756, 2001.

[2] B. Dunn, H. Kamath, and J. M. Tarascon, "Electrical energy storage for the grid: A battery of choices," Science, vol. 334, no. 6058, pp. 928-935, 2011.

[3] H.-I. Su and A. E. Gamal, "Modeling and analysis of the role of fastresponse energy storage in the smart grid," Proceedings of the FortyNinth Annual Allerton Conference on Communication, Control, and Computing, Illinois, USA, pp. 719-726, 2011.

[4] J. Eyer and G. Corey, Energy Storage for the Electricity Grid: Benefits and Market Potential Assessment Guide. SANDIA Report, 2010.

[5] J. P. Barton and D. G. Infield, "Energy storage and its use with intermittent renewable energy," IEEE Transactions On Energy Conversion, vol. 19, no. 2, pp. 441-448, 2004.

[6] P. D. Brown, J. A. P. Lopes, and M. A. Matos, "Optimization of pumped storage capacity in an isolated power system with large renewable penetration," IEEE Transactions on Power Systems, vol. 23, no. 2, pp. 523-531, 2008.

[7] J. G. Gonzalez, R. M. R. de la Muela, L. M. Santos, and A. M. Gonzalez, "Stochastic joint optimization of wind generation and pumped-storage units in an electricity market," IEEE Transactions on Power Systems, vol. 23, no. 2, pp. 460-468, 2008.

[8] N. Löhndorf and S. Minner, "Optimal day-ahead trading and storage of renewable energies: an approximate dynamic programming approach," Energy Systems, vol. 1, pp. 61-77, 2010.

[9] R. Carmona and M. Ludkovski, "Valuation of energy storage: An optimal switching approach," Quantitative Finance, vol. 10, no. 4, pp. 359-374, 2010.

[10] J. H. Kim and W. B. Powell, "Optimal energy commitments with storage and intermittent supply," Operations Research, vol. 59, no. 6, pp. 13471360, 2011.

[11] P. Harsha and M. Dahleh, "Optimal management and sizing of energy storage under dynamic pricing for the efficient integration of renewable energy," submitted, pp. 1-34, 2012.

[12] H. Pandzic, J. M. Morales, A. J. Conejo, and I. Kuzle, "Offering model for a virtual power plant based on stochastic programming," Applied Energy, vol. 105, pp. 282-292, 2013.

[13] H. Pandzic, I. Kuzle, and T. Capuder, "Virtual power plant mid-term dispatch optimization," Applied Energy, vol. 101, pp. 134-141, 2013.

[14] A. Eydeland and K. Wolyniec, Energy and Power Risk Management: New Developments in Modeling, Pricing, and Hedging. Wiley; 1 edition, 2002

[15] T. Kanamura and K. Ōhashi, "A structural model for electricity prices with spikes: Measurement of spike risk and optimal policies for hydropower plant operation," Energy Economics, vol. 29, no. 5, pp. 10101032, 2007.

[16] M. Burger, B. Graeber, and G. Schindlmayr, Managing Energy Risk: An Integrated View on Power and Other Energy Markets, 1st ed. Wiley, 2007.

[17] Z. Hameed, J. Vatn, and J. Heggset, "Challenges in the reliability and maintainability data collection for offshore wind turbines," Renewable Energy, vol. 36, pp. 2154-2165, 2011.
[18] US Department of Energy Report, "U.S. wind energy production and manufacturing reaches record highs," pp. 1-3, 2013.

[19] P. Artzner, F. Delbaen, J. Eber, and D. Heath, "Coherent measures of risk," Mathematical Finance, vol. 9, pp. 203-228, 1999.

[20] G. C. Pflug and W. Romisch, Modeling, Measuring and Managing Risk. World Scientific Publishing Company, August 2007.

[21] H. Follmer and A. Schied, Stochastic Finance: An Introduction in Discrete Time. De Gruyter; 3rd Edition, January 2011.

[22] A. Downward, D. Young, and G. Zakeri, "Electricity contracting and policy choices under risk-aversion," submitted to Operations Research, pp. 1-43, 2012.

[23] M. Carrión, A. B. Philpott, A. J. Conejo, and J. M. Arroyo, "A stochastic programming approach to electric energy procurement for large consumers," IEEE Transactions on Power Systems, vol. 22, no. 2, pp. 744-754, 2007.

[24] A. Cartea and M. Figueroa, "Pricing in electricity markets: a mean reverting jump diffusion model with seasonality," Applied Mathematical Finance, vol. 12, no. 4, pp. 313-335, 2005.

[25] H. Geman and A. Roncoroni, "Understanding the fine structure of electricity prices," Journal of Business, vol. 79, no. 3, pp. 1225-1261, 2006.

[26] J. Hinman and E. Hickey, "Modeling and forecasting short-term electricity load using regression analysis," Institute of Regulatory Policy Studies, pp. $1-51,2009$.

[27] L. J. Soares and M. C. Medeiros, "Modeling and forecasting shortterm electricity load: A comparison of methods with an application to Brazilian data," International Journal of Forecasting, vol. 24, no. 4, pp. 630-644, 2008

[28] T. Ackermann, Wind Power in Power Systems, second edition ed. Wiley, May 2012.

[29] C. Carrillo, A. F. Obando Montaño, J. Cidrás, and E. Díaz-Dorado, "Review of power curve modelling for wind turbines," Renewable and Sustainable Energy Reviews, vol. 21, pp. 572-581, 2013.

[30] D. J. MacKay, Sustainable Energy-Without the Hot Air. UIT Cambridge Ltd.; 1 edition, 2009.

[31] D. C. Hill, D. McMillan, K. R. W. Bell, and D. Infield, "Application of auto-regressive models to U.K. wind speed data for power system impact studies," IEEE Transactions on Sustainable Energy, vol. 3, no. 1, pp. 134-141, 2012.

[32] T. Burton, D. Sharp, N. Jenkins, and E. Bossanyi, Wind Energy Handbook. Wiley, June 2011.

[33] P. M. van de Ven, N. Hegde, L. Massoulie, and T. Salonidis, "Optimal control of end-user energy storage," arXiv:1203.1891, pp. 1-9, 2012.

[34] D. Duffie and J. Pan, "An overview of value at risk," Journal of Derivatives, vol. 4, no. 3, pp. 7-49, 1997.

[35] R. T. Rockafellar and S. Uryasev, "Optimization of conditional valueat-risk," The Journal of Risk, vol. 2, no. 3, pp. 21-41, 2000.

[36] S. Alexander, T. F. Coleman, and Y. Li, "Minimizing VaR and CVaR for a portfolio of derivatives," Journal of Banking and Finance, vol. 30, no. 2, pp. 583-605, 2006

[37] H. Xu and D. Zhang, "Smooth sample average approximation of stationary points in nonsmooth stochastic optimization and applications," Mathematical Programming, vol. 119, pp. 371-401, 2009.

[38] J. Nocedal and S. Wright, Numerical Optimization. Springer; 2nd edition, July 2006.

[39] T. F. Coleman and Y. Li, "An interior trust region approach for nonlinear minimization subject to bounds," SIAM Journal on Optimization, vol. 6 , no. 2 , pp. $418-445,1996$

[40] A. Holder, "Parametric LP analysis," in Wiley Encyclopedia of Operations Research and Management Science, J. Cochran, Ed. John Wiley \& Sons, 2010.

[41] A. Nemirovski and A. Shapiro, "Convex approximations of chance constrained programs," SIAM Journal on Optimization, vol. 17, no. 4, pp. 969-996, 2006.

[42] A. Ben-Tal, L. El Ghaoui, and A. Nemirovski, Robust Optimization. Princeton University Press, 2009.

[43] D. Bertsimas, D. B. Brown, and C. Caramanis, "Theory and applications of robust optimization," SIAM Review, vol. 53, no. 3, pp. 464-501, 2011.

[44] F. Babonneau, J. P. Vial, and R. Apparigliato, "Robust optimization for environmental and energy planning," in Handbook on Uncertainty and Environmental Decision Making, J. A. Filar and A. Haurie, Eds. International Series in Operations Research and Management Science, Springer Verlag, 2010, pp. 79-126.

[45] A. H. Hajimiragha, C. A. Canizares, M. W. Fowler, S. Moazeni, and A. Elkamel, "A robust optimization approach for planning the transition to plug-in hybrid electric vehicles," IEEE Transactions On Power Systems, vol. 26, no. 4, pp. 2264-2274, November 2011 
Somayeh Moazeni (M'13) received her BSc (2004) and MSc (2005) in mathematics from Tehran Polytechnic, Tehran, Iran, MMATH (2006) in combinatorics and optimization from the University of Waterloo, Ontario, Canada, $\mathrm{Ph} . \mathrm{D}$ (2011) in computer science from the University of Waterloo, Ontario, Canada. She is currently a postdoctoral research associate in the Department of Operations Research and Financial Engineering in Princeton University, New Jersey, USA. Her research interest includes numerical optimization, optimization under uncertainty especially stochastic optimization and robust optimization, risk management, and financial optimization. She has been the recipient of several national and international awards such as the 2009 Google Canada Anita Borg Memorial Scholarship and the 2013 NSERC (Natural Sciences and Engineering Research Council of Canada) Postdoctoral Fellowship.

Warren B. Powell (M'06) is a Professor in the Department of Operations Research and Financial Engineering at Princeton University, New Jersey, USA, where he has taught for over 30 years. He is the director of the ComputAtional STochastic optimization and LEarning (CASTLE) Laboratory and the Princeton laboratory for ENergy Systems Analysis (PENSA) focusing on energy systems analysis, computational stochastic optimization and learning. He is the author of the book "Approximate Dynamic Programming: Solving the curses of dimensionality", co-author of "Optimal Learning", and co-editor of "Learning and Approximate Dynamic Programming: Scaling up to the Real World". He is the recipient of Docteur Honoris Causa from the University of Quebec in Montreal, Canada, in 2013, winner of the 2009 Daniel Wagner Prize for extending approximate dynamic programming to very high-dimensional problems for Schneider National, and many other prizes such as Informs Fellows Award, Presidential Young Investigator Award, and various best paper prizes.

Amir H. Hajimiragha (S'09-M'10-SM'13) received his BSc (1995) from K. N. Toosi University of Technology, Tehran, Iran, MSc (2005) from Royal Institute of Technology (KTH), Stockholm, Sweden, and PhD (2010) from the University of Waterloo, Waterloo, Ontario, Canada, all in Electrical Engineering. He began his career in 1996 as a research engineer at the Electric Power Research Center (EPRC), Tehran, Iran, where he worked on flicker assessment of alloy steel plants. Between 1998 and 2003 he worked at Niroo Research Institute (NRI), also in Tehran, Iran in different research and managerial positions, including the fields of power quality, energy management, power plants, and scientific publishing. Prior to joining General Electric (GE) in 2010, he worked as a PostDoc researcher at the University of Waterloo, where he investigated the grid impacts of fuel cell and plug-in hybrid electric vehicles. Currently, he is a lead application engineer at the GE GridIQ Innovation Center, Markham, Ontario, Canada. Here he is involved in research and development and new product introduction (NPI) programs concerning microgrids and distribution systems automation. He has been the recipient of several national and international awards, including the 2011 IET's international innovation award in the category of "Built Environment", the 2008 MITACS (Mathematics of Information Technology and Complex Systems) National Award in the Category of "Best Novel Use of Mathematics in Technology Transfer", and the 2008 University of Waterloo Exceptional Teaching Award. His research interests are in the areas of integrated energy systems, hydrogen economy, microgrids, grid impacts of alternative-fuel vehicles, and smart grids. He is a senior member of the IEEE, a member of the IEEE microgrid control task force and a member of the IEEE 1547 working group. 